\title{
The Hemophilic Joints
}

E. Carlos Rodriguez-Merchan*

Department of Orthopaedic Surgery, La Paz University Hospital, Madrid, Spain; and School of Medicine, Autonomous University, Madrid, Spain

\begin{abstract}
Recurrent hemarthroses in childhhood before the initiation of primary prophylaxis will result in joint damage after a decade or later. The best way that we have today to protecting against hemophilic arthropathy is primary prophylaxis. The decision to institute early full prophylaxis by means of a port (central venous access device) has to be balanced against the child's bleeding tendency, the family's social situation and the experience of the specific hemophilia center. The reported complication rates for infection and thrombosis have varied considerably from center to center. Risk of infection can be reduced by repeated education of patients and staff, effective surveillance routines and limitations on the number of individuals allowed to use the device. In discussing options for early therapy, the risks and benefits should be thoroughly discussed with the parents. From a practical point of view, primary prophylaxis together with synovectomy (radioactive, chemical, arthroscopic, or open) to avoid joint bleeding, can help halt hemophilic synovitis. Radiosynovectomy is a relatively simple, virtually painless and inexpensive treatment for chronic hemophilic synovitis, even in patients with inhibitors and must be the best choice for patients with persistent synovitis (which must be confirmed by US and/or MRI). Arthroscopic joint debridement may be indicated in younger hemophiliacs when there is relative preservation of the joint architecture and congruity, after the failure of three radiosynovectomies with a 6-month interval, and when joint replacement is not considered to be a viable alternative. Total joint arthroplasty should be indicated in hemophiliacs with severe joint pain and disability, including those who are HIV-positive. Hemophilia is a risk factor for infection after total joint arthroplasty. An extended period (2-3 weeks) of adequate hemostatic function is necessary to achieve normal healing after orthopedic procedures in hemophilia.
\end{abstract}

Keywords: Hemophilia; Joints; Management

\section{Introduction}

It is well known that about $90 \%$ of people with severe hemophilia experience chronic degenerative changes (hemophilic arthropathy) in one to six major joints (ankles, elbows, knees) by the second or third decade of life. Such degenerative changes are mainly due to spontaneous recurrent intra-articular hemorrhages that persons with hemophilia frequently present. A critical factor for avoiding hemophilic arthropathy is the prevention of articular hemorrhages by means of prophylactic treatment. However, despite regular infusions of anti-hemophilic concentrate at an early age (prophylactic treatment), recurrent hemarthrosis and the possibility of hemophilic arthropathy still persist in some patients (subclinical haemorrhages). On the other hand, the pathogenesis of the progression from recurrent hemarthrosis to hemophilic arthropathy, in particular in the early stages, is incompletely understood [1].

The best way that we have today to protecting against hemophilic arthropathy (cartilage damage) is primary prophylaxis (from cradle to college). Starting prophylaxis gradually with once-weekly injections has the presumed advantage of avoiding use of a central venous access device, such as a Port A Cath, which is often necessary for frequent injections in very young boys. The decision to institute early full prophylaxis by means of a port has to be balanced against the child's bleeding tendency, the family's social situation and the experience of the specific hemophilia center. The reported complication rates for infection and thrombosis have varied considerably from center to center. Risk of infection can be reduced by repeated education of patients and staff, effective surveillance routines and limitations on the number of individuals allowed to use the device. In discussing options for early therapy, the risks and benefits should be thoroughly discussed with the parents. For children with inhibitors needing daily infusions for immune tolerance induction, a central venous line is often unavoidable and is associated with an increased incidence of infections $[2,3]$.

Any preventive measure should be implemented very early, because a relatively short exposure of cartilage to blood may result in longlasting changes in chondrocyte metabolism that may eventually lead to chronic degenerative changes (hemophilic arthropathy). There are three basic invasive methods for the management of the hemophilic joint: synovectomy (radioactive, chemical, arthroscopic or open), arthroscopic joint debridement and joint arthroplasty [4].

Other conservative treatments such as physiotherapy and/or sports therapy as well as other fields of rehabilitation medicine are not focussed in this paper. In other words, this article only deals with orthopaedic treatment options. In this paper current knowledge on the pathogenesis, diagnosis and orthopaedic treatment of the hemophilic joint will be revised.

\section{Pathogenesis}

Hemophilic arthropathy is characterized by chronic proliferative synovitis and cartilage destruction. Both events are the consequence of recurrent intra-articular bleeding. However, their exact pathogenesis is still poorly understood. In vitro studies have shown that a four-day

*Corresponding author: Prof. E. Carlos Rodriguez-Merchan, Department of Orthopaedic Surgery, La Paz University Hospital, Paseo de la Castellana 261 28046-Madrid, Spain, Fax: +34-91-5712871; E-mail: ecrmerchan@gmx.es

Received October 24, 2011; Accepted November 15, 2011; Published November 18, 2011

Citation: Rodriguez-Merchan EC (2011) The Hemophilic Joints. J Blood Disord Transfus 2:111. doi:10.4172/2155-9864.1000111

Copyright: () 2011 Rodriguez-Merchan EC. This is an open-access article distributed under the terms of the Creative Commons Attribution License, which permits unrestricted use, distribution, and reproduction in any medium, provided the original author and source are credited. 
duration of blood exposure produces a blood concentration- and timedependent inhibition of cartilage matrix formation and an increases release of matrix components, both events resulting in a loss of matrix [5].

The experimental model of hemophilic synovitis in the mouse from a Chicago group [6,7] have shown that after 14 days of a major joint hemorrhage, the massive swelling of the joint resolves but the tissues are brown with hemosiderin staining and the joint cavity is filled with a dense inflammatory cell infiltrate. Vascular hyperplasia is also evident. The articular surface is irregular with pannus formation and the underlying bone is dysmorphic. After 30 days, there is marked cartilage and subchondral bone erosion.

Three important papers [8-10] studied the pathogenesis of synovitis and cartilage alterations in haemophilic joints from the experimental point of view. In the first article, Hooiveld et al investigated the effect of a limited number of joint bleedings, combined with loading of the affected joint, in the development of progressive degenerative joint damage [8]. They concluded that experimental joint bleedings, when combined with loading of the involved joint, result in features of progressive degenerative joint damage, whereas similar joint hemorrhages without joint loading do not. The authors suggest that this might reflect a possible mechanism of joint damage in hemophilia.

In the second paper, Hakobyan et al analyzed the pathogenesis of hemophilic synovitis in a murine model of human haemophilia A [9]. Hemarthrosis resulted in pathologic changes in human hemophilic synovitis and a marked increase in synovial proliferation. In vitro, iron induced the expression of the $\mathrm{p}-53$ binding protein $\mathrm{mdm} 2$ by normal human synovial cells approximately 8 -fold. The authors concluded that molecular changes induced by iron in the blood may be the basis of the increase in cell proliferation and the development of hemophilic synovitis.

In the third article, Valentino et al studied hemophilic synovitis. They developed a murine model of human factor VIII deficiency, which facilitates the examination of large number of animals and tissue specimens [10]. They showed that controlled, blunt trauma to the joint consistently resulted in joint swelling, because of a combination of bleeding and inflammation.

Despite the fact that these three papers are very interesting with regard to the understanding of hemophilic synovitis and the initial stages of cartilage damage in hemophilic joints, the link between both phenomena, is still very poorly understood. In fact, previous studies have shown that synovitis is not primarily responsible for the cartilage damage after intra-articular bleeding, but rather that the exposure of cartilage to blood induces direct adverse effects. The data presented by Hooiveld et al demonstrate that a limited series of bleedings with loading of the involved joint adversely influences cartilage matrix turnover and integrity. In addition, increased (although still very mild) synovial inflammation was found in the experimental joints. The changes in chondrocyte activity are characteristics of early osteoarthritic cartilage. The clinical relevance of these data appear to suggest that avoiding or minimizing joint loading might help protecting against hemophilic arthropathy.

Hakobyan et al demonstrated that blood induces synovitis and mdm2 expression in a murine model of human hemophilia [9]. They have provided evidence that iron may be linked to multiple molecular changes (c-myc and $\mathrm{mdm} 2$ ), resulting in the pathologic proliferative changes observed in hemophilic synovitis. They speculated that iron is responsible for the aberrant gene expression. An increase in mdm2 expression decreases p53 activity, resulting in abrogation of synovial cells apoptosis and/or an increase in proliferation. The increase in c-myc further drives synovial cell proliferation. The identification of $\mathrm{mdm} 2$ as a key mediator involved in haemophilic synovitis suggests that this molecule (or its pathway) may be a target for future therapeutic interventions. From a practical point of view, radiosynovectomy, together with primary prophylaxis to avoid joint bleeding, can help halt hemophilic synovitis. Ideally, however, radiosynovectomy should be performed before the articular cartilage has eroded. Radiosynovectomy is a relatively simple, virtually painless and inexpensive treatment for chronic hemophilic synovitis, even in patients with inhibitors and is the best choice for patients with persistent synovitis.

Valentino et al described a novel murine model of human haemophilia A, and the effects of hemarthrosis on the physical, gross and microscopic changes evoked following joint bleeding [10] Hemosiderin was found in the synovial membrane after provoking a controlled blunt trauma to the knee joint. Similar to hemarthrosis in human haemophilia, joint bleeding resulted in acute morbidity evidenced by inactivity, weight loss and immobility. Valentino et al's murine model appears to be of use in studying the pathogenesis of hemophilic synovitis and for testing potential therapeutic interventions.

The three reviewed papers are very important for a better understanding of the pathogenesis of hemophilic synovitis and arthropathy. These papers try to shed light on the molecular mechanisms behind the hemophilic arthropathy, so that their clinical relevance may become important in the near future. From the clinical point of view, primary prophylaxis, arthroscopic synovectomy are the best ways that we have today of protecting against hemophilic synovitis and arthropathy.

\section{Diagnosis by Imaging}

Both prophylaxis and radiosynovectomy would require MRI (magnetic resonance imaging) for detecting synovitis, because it is more sensitive than clinical examination or plain radiographs. However, MRI is expensive and requires sedation in younger children. The article of Acharya et al has compared ultrasonography (USG) and the current gold standard, MRI, in the diagnosis of haemophilic synovitis [11]. According to Acharya et al USG-PDS (power Doppler sonography) appears to be an inexpensive and easily implemented imaging tool for the diagnosis of hemophilic synovitis [11]. Moreover, it could be useful in tailoring effective primary prophylaxis and in confirming the diagnosis of synovitis in an objective way before indicating a radiosynovectomy. Multi-centre studies are required to fully validate USG as an important diagnostic tool, which was used in persons with hemophilia in my center for the first time in the early 1990s.

Previous studies, the first one published in 1992, have suggested the potential of USG in the assessment of hemophilic arthropathy, although standardization and validation of USG were not established [12-14].

In a pilot study performed by Acharya et al, thirty-one subjects including 33 joints (ankles, elbows, knees) underwent dynamic contrast enhanced (DCE)-MRI and USG-PDS [11]. Measurements of synovial thickening and synovial vascularity correlated strongly with those obtained with DCE-MRI.

Acharya et al have suggested that USG with power Doppler supplementation may be a user-friendly alternative to MRI. Its authors favoured USG because it is cheaper, more readily available than MRI, and more easily tailored to examination of multiple joints in the same 
day [11]. The low cost of this imaging tool ( $\$ 450$ USG-PDS versus $\$ 1800 \mathrm{MRI}$ ) without a need for sedation in young children and easier contralateral correlation make it very attractive for them.

There is a need for further standardization and validation in multi-centre studies to ensure accurate diagnosis, reproducibility and reliability before USG-PDS can be an established method in hemophilia diagnostics. In summary, USG could be useful in tailoring effective primary prophylaxis and in confirming the diagnosis of synovitis [15].

\section{Treatment}

COX-2 Inhibitors: Rattray et al suggested that celecoxib is effective in the management of hemophilic synovitis, target joints and pain. However, the authors recognise several important limitations in their study: its retrospective nature, the lack of control group, the small number of patients, the variable dosing of celecoxib and factor replacement, the inclusion of inhibitor patients, and the use of nonvalidated outcome scales. Moreover, the clearly state that controlled clinical trials are required to confirm their findings [16].

On the other hand the authors state that a trial of celecoxib could also be considered in an attempt to obviate the need for either radiosynovectomy or arthroscopic synovectomy. My view is that a lot of work remains to be done to confirm the little clinical experience reported in this paper, that is to say, the role of celecoxib in the treatment of haemophilic synovitis, target joints and pain in adults and children with hemophilia. Moreover, today it is hard to believe that celecoxib could be an alternative to radiosynovectomy, as the authors comment.

The authors of the article have treated 12 patients between 9 and 54 years of age with celecoxib for a total of 14 courses of celecoxib treatment. They have used celecoxib for three different indications: chronic synovitis (8 patients), pain (3 patients) and target joint (1 patient). In the synovitis cases, a response was noted in seven of eight patients. In the 3 cases in which celecoxib was used for pain, a response was noted in all patients. However, no response was noted in the patient with the target joint. On the other hand, no serious adverse effects including hypertension were observed. Efficacy in chronic synovitis and pain was judged subjectivelly as effective, partially effective or ineffective. Efficacy in resolution of the target joint was judged as effective if the target joint resolved or ineffective if it did not resolve.

On the other hand the authors feel that it is safe to treat patients with hemophilia with COX-2 inhibitors provided that they do not have other risk factors for cardiovascular disease or the potential benefits outweigh the risks for a cardiovascular event and that their blood pressure is monitored on a regular basis. That is to say, celecoxib has some potential limitations for its use in hemophilia despite in the study no adverse events were noted including hypertension or other cardiovascular events. In the study dosing regimens were chosen empirically. Doses were prescribed as $100 \mathrm{mg}$ twice daily, $200 \mathrm{mg}$ daily and $200 \mathrm{mg}$ twice daily. Dosing ranged from 1.01 to $3.83 \mathrm{mg} \mathrm{kg}-1$ with a mean of $2.56 \mathrm{mg} \mathrm{kg}-1$. For pain, patients were treated as needed, for chronic synovitis, patients were treated daily for 30 days and continued indefinitely if improvement was noted. For target joints, patients were treated until resolution of the target joint or until therapy was deemed ineffective.

\section{Radiosynovectomy}

Regarding the treatment of chronic synovitis, radiosynovectomy has been reported to be effective. Moreover, its long-term safety has been established after 35 years of using radiosynovectomy worldwide, with no damage reported in relation to the radioactive materials [1719]. The general recommendation is to use 90Yttrium or ${ }^{186} \mathrm{Rhenium}$ synovectomy, because these agents have proved to be efficient for the treatment of chronic hemophilic synovitis, even in patients with inhibitors [20-22].

Mortazavi et al reported on the treatments results of radiosynovectomy with $32 \mathrm{P}$ chromic phosphate with emphasis on clinical aspects [23]. In most of the injected joints, the range of motion remained stable or improved. For the authors of the paper an early radiosynovectomy might be more helpful in terms of stability of response to treatment. Mortazavi et al demonstrated once more that radiosynovectomy is a useful procedure for the treatment of chronic hemophilic synovitis. In fact, they have confirmed what many authors have proved many years before: that radiosynovectomy is an excellent procedure for the management of chronic synovitis in persons with hemophilia.

On the other hand, it is important to emphasize that controversy exists regarding which type of synovectomy is better. Most authors in developed countries use radiosynovectomy $\left({ }^{90} \mathrm{Yttrium},{ }^{186} \mathrm{Rh}\right.$ henium) [20], while others utilize chemical synovectomy mainly because of the lack of availability of radioactive materials. The general recommendation is to use ${ }^{90}$ Yttrium or ${ }^{186} \mathrm{Rhenium}$ synovectomies, because these agents have proved to be efficient for the treatment of chronic hemophilic synovitis, even in patients with inhibitors. Moreover, no complications related to the use of radioactive materials have been reported after 30 years of being used worldwide [21].

Let me say that the experience at my Center made us to empirically stop using ${ }^{90}$ Yttrium for the small joints (elbows and ankles) four years ago. The reason was that ${ }^{186}$ Rhenium appeared to be more effective than ${ }^{90}$ Yttrium in reducing hemarthroses in such small joints. On the other hand, we also observed that for the knees ${ }^{90}$ Ytrium appeared to be more effective. Therefore, nowadays we are using ${ }^{186}$ Rhenium for the elbows and ankles, and ${ }^{90}$ Yttrium for the knees. Nonetheless, I do not know which radioactive material is better. Only prospective comparative studies at different joints, with different materials could clarify such a controversy.

My opinion is that we must perform early radiosynovectomies (irrespective of the age of the patient), that is to say, as soon as synovitis is diagnosed. Then, my current recommendation is to use ${ }^{90}$ Yttrium for the knees. Nonetheless, despite the controversies previously mentioned, there is no doubt that radiosynovectomy is an excellent, cheap, easy ad safe procedure for the treatment of hemophilic synovitis that I highly recommend and use since more than 35 years ago.

Kavakli et al reported the results of synovectomy performed with ${ }^{186}$ Rhenium $\left({ }^{186} \mathrm{Rh}\right)$ [24]. The authors injected $2 \mathrm{mCi}(74 \mathrm{MBq})$ of ${ }^{186}$ Rhenium in all the treated joints. Their results confirm those previously published by others on the value of ${ }^{186} \mathrm{Rh}$ radiosynovectomy in medium-sized joints in hemophilia patients. In this article, Kavakli et al demonstrate once more that radiosynovectomy is a useful procedure for the treatment of chronic hemophilic synovitis. In fact, they have confirmed what many authors have proved many years before: that radiosynovectomy is an excellent procedure for the management of chronic synovitis in patients with hemophilia. The results of Kavakli et al confirm those previously published in Madrid on the value of ${ }^{186}$ Rhenium synoviorthesis in medium-sized joints in 
hemophilia patients suffering from chronic synovitis [25]. Moreover, no complications related to the use of radioactive materials have been reported after more than 35 years of being used worldwide.

\section{Chemical Synovectomy}

Two interesting papers focused on the treatment of chronic hemophilic synovitis by means of chemical synovectomy [26,27]. Corrigan et al have used oral D-penicillamine for the treatment of 16 patients. The drug was given as a single dose in the morning before breakfast [26]. The dose was $5-10 \mathrm{mg} \mathrm{kg}^{-1}$ bodyweight, not to exceed $10 \mathrm{mg} \mathrm{kg-}^{-1}$ in children, or $750 \mathrm{mg}$ per day in adults. The duration of treatment was 2 months to 1 year (median 3 months). Ten patients had an unequivocal response, three had a reduction in palpable synovium and three had no response. Minor reversible drug side-effects occurred in two patients (proteinuria in one and a rash in the second).

Concerning the first article, Corrigan et al previously reported in a preliminary study that $\mathrm{D}$-penicillamine is effective in controlling hemophilic synovitis [26]. The ages of the 16 patients studied ranged from 6 years to 55 years (median 14 years). The mechanism by which D-penicillamine exerts its anti-inflammatory effect is not known. The study of Corrigan et al has two main limitations: the small number of patients, and the lack of use of ultrasound and/or magnetic resonance imaging (MRI) for diagnostic purposes. It is also important to emphasize two potential side effects of D-penicillamine: aplastic anemia and renal disease. To minimize the possibility of side-effects, Corrigan et al have suggested that the drug be used on a short-term basis (i.e. 3-6 months) and the amount be restricted to $5-10 \mathrm{mg} \mathrm{kg-}_{-}{ }^{1}$ for children and less than $750 \mathrm{mg}$ day- ${ }^{-1}$ for adults, given as one dose in the morning at least 1 hour before eating.

I agree with the authors' statement that radiosynovectomy using intra-articular ${ }^{90} Y$ Ytrium or ${ }^{32} \mathrm{Rhenium}$ has been reported to be effective; however, I disagree with the authors' comment that this is an invasive procedure whose long-term safety has not been established. In fact, the long-term safety has been established after 30 years of using radiosynovectomy worldwide, with no damage reported in relation to the radioactive materials [20]. Corrigan et al also state that the results of their study suggest that D-penicillamin is an effective drug for the treatment of hemophilic synovitis. However, further clinical trials should be performed due to the limitations of their study (small number of patients and lack of use of diagnostic techniques such as ultrasound and/or MRI). Finally, I also disagree with their statement that the drug could be particularly useful in high-responding inhibitor patients who are not amenable to invasive therapeutic modalities. It has been shown that patients with inhibitors can undergo radiosynovectomy with excellent results [28].

Regarding the second article [27], it is important to emphasize that controversy exists regarding which type of medical synovectomy is better. Most authors in developed countries use radiosynovectomy ${ }^{(90}$ Yttrium, 32Phosphorus) [21], while others utilize chemical synovectomy mainly because of the lack of availability of radioactive materials [29]. My view is that further studies with an adequate number of patients and an appropriate follow-up are needed to confirm the efficacy of oral penicillamine and rifampicin synovectomy for chronic hemophilic synovitis. In other words, the aforementioned papers are preliminary studies requiring confirmation. Meanwhile, the general recommendation is to use 90Yttrium or 32Phosporus radiosynovectomies, because these agents have proved to be efficient for the treatment of chronic hemophilic synovitis, even in patients with inhibitors. Moreover, no complications related to the use of radioactive materials have been reported after 30 years of being used worldwide [21].

\section{Arthroscopic Joint Debridement}

Arthroscopic joint debridement may be indicated in younger haemophiliacs when there is relative preservation of the joint architecture and congruity, when joint replacement is not considered to be a viable alternative. Joint debridement is frequently performed in combination with synovectomy. Between the second and fourth decades, many haemophilic patients develop joint destruction (arthropathy). At this stage possible treatments include arthroscopic joint debridement and . Debridement should be considered in the young haemophiliac to avoid, or delay, total joint arthroplasty. The operation may give the patient years of life without pain and it appears to slow the development of radiographic changes [30].

\section{Total Joint Arthroplasty}

The most frequent joint arthroplasty performed on hemophilic patients is total knee arthroplasty (TKA). Arthroplasty of other joints (hip, shoulder, elbow, ankle) are very uncommon.

Sheth et al performed a single centre study to assess the results of total knee arthroplasty (TKA) in hemophilia. The main conclusions were that TKA is an effective treatment for advanced hemophilic arthropathy and that HIV-infected persons should not be denied TKA on the basis of fear of infection or mortality [31]

TKA should be indicated in hemophiliacs with severe knee pain and disability, including those who are HIV-positive. However, the orthopaedic surgeon should weigh up the risks and benefits, particularly in HIV-positive patients [32,33].

Norian et al reported that TKA performed to treat hemophilic arthropathy has a high risk of failure as a result of infection. It may be difficult to salvage a prosthesis complicated by infection. However, the life expectancy of hemophilic patients is lower than that of the general population of patients treated with TKA, and the improvement in the quality of life after TKA for hemophilic arthropathy may outweigh the risk of failure [34]. In Norian's et al article no difference was found in the CD4 lymphocyte count between patients in whom infection developed and those in whom it did not [34]. These findings contrast with the general belief among orthopaedic surgeons treating hemophiliacs, which is that those HIV-positive haemophiliacs with a CD4 count $<200 / \mathrm{mm}^{3}$ have a higher risk of infection than those who are HIV-negative or HIV-positive but with a CD4 count $>200 / \mathrm{mm}^{3}$ [31].

Ragni et al [35] found that postoperative infection developed in ten (15\%) of sixty-six seropositive hemophiliacs with a CD4 lymphocyte count of $<200 / \mathrm{mm}^{3}$. However, they did not include a control group with CD4 lymphocyte counts of $>200 / \mathrm{mm}^{3}$. Ragni's et al $15 \%$ infection rate is similar to Norian et al $13 \%$ rate in patients with an average CD4 lymphocyte count of $<200 / \mathrm{mm}^{3}$. This comparison supports the conclusion that hemophilia is a risk factor for infection independent of CD4 lymphocyte count [35].

My view is that hemophilia per se is a risk factor for infection (risk related to the disease), but also that a CD4 count $<200 / \mathrm{mm}^{3}$ significantly increase the risk of infection after TKA (risk related to severe immunodeficiency) [32]. The outcome of the infections at the sites of the TKAs is generally poor. In fact, in Norian's et al series, of the seven patients in whom infection developed, only two ultimately 
had a successful functioning knee prosthesis, and one of them required long-term suppressive antibiotic therapy [34]. Regarding prosthetic survival, it commonly is in the range of $90 \%$ to $95 \%$ after ten to fifteen years in patients with osteoarthritis. The results of Norian et al indicate that prosthetic survival in patients with hemophilic arthropathy is less favorable, and therefore, patients should be counseled about the limited longevity of the implant and the relatively high risk of failure or poor outcome due to infection.

A multicentre review has shown that total knee replacement for advanced hemophilic arthropathy has good and excellent results in about $85 \%$ of cases. The principle risk is late infection, which can occur regardless of HIV status. However, this risk appears increased in the patient with CD4 count under $200 / \mathrm{mm}^{3}$. It should not be inferred that a TKR should be avoided in an HIV-positive haemophilia patient today, but that the orthopaedic surgeon, treatment team, and the patient should weigh the risks and benefits carefully [31].

Powell et al evaluated the incidence of deep infection rates following total knee and hip arthroplasties in HIV-seropositive and HIV-seronegative persons with hemophilia [36]. Rate of primary joint infection per artificial joint-year by HIV status was compared by Poisson regression. Deep infections developed in five (9.8\%) of 51 replacement joints. The incidence rate of joint infection ( $0.98 \mathrm{vs}, 1.46$ per 100 joint-years) was not increased with HIV (relative risk, RR: 1.49, 95\% CI: 0,25-8,93, $\mathrm{p}=0,66$ ).

Total joint replacement is an option for the management of advanced hemophilic arthropathy of the hip and knee [31,32]. On the other hand, the surgical techniques of total knee arthroplasty (TKA) in hemophiliacs is technically challenging. Therefore, the risk of postoperative complications is high, including deep infection and joint loosening. Those with human immunodeficiency infection (HIV), especially those with CD 4 cell counts $<200$ cells $\mathrm{mm}^{-}{ }^{3}$, could have infection rates much higher than patients withouth HIV infection [34].

Despite the wide variety of prostheses and postoperative protocols, TKA and THA deep infection rate in the general population is about $1-2 \%$. However, infections rates in hemophilic patients are much higher, ranging from $7 \%$ to $26.5 \%$.

Powell et al have evaluated the results of TKAs and THAs in a group of 32 persons with hemophilia (51 primary arthroplasties) [36]. Deep infections occurred in five patients after 51 primary replacements (9.8\%), four after TKAs and one following a THA. Their infection rate of $9.8 \%$ following primary total joint replacement in persons with hemophilia irrespective of HIV status is comparable with previously reported rates.

Prosthetic survival after TKA in patients with osteoarthritis or rheumatoid arthritis is about $90 \%$ after $10-15$ years. Joint survivorship in persons with hemophilia is about $90 \%$ after 5 years and $72 \%$ after 10 years. The low rate of joint survival in Powell et al's article (69\% at 5 years and $29 \%$ at 10 years) was due to deaths from HIV-related opportunistic infections of liver failure [36]. Additional studies are needed to determine whether the results of Powell et al's can be generalized to other HIV-positive risk groups undergoing knee or hip replacement arthroplasties.

It is noteworthy that all the infections in the study of Powell et al occurred before the use of highly active antiretroviral therapy (HAART), which became available for general use in the mid-1990s. HAART had led to durable viral load suppression and immune reconstitution in many patients. Although persons with hemophilia are intrinsically at higher risk for joint infection than the general orthopaedic patient following total joint replacement, Powell et al believe that TJR is a reasonable option for those who are suffering from severe hemophilic arthropathy, regardless of their HIV status.

It has been reported that healing of skin wounds is delayed in hemophilia $\mathrm{B}(\mathrm{HB})$ mice $[37,38]$. HB mice do not bleed excessively at wounding, yet rebleed hours to days later. McDonald et al reported that wounds show abnormal histology: s.c. hematoma formation; delayed re-epithelialization; delayed macrophage influx; and an increase in wound site angiogenesis. Therefore, it seems that an extended period of adequate hemostatic function is necessary to achieve normal healing, probably because the risk of hemorrhage is increased by vascular remodeling and angiogenesis during the healing process.

\section{Conclusions}

Major hemarthroses must be treated as soon as possible (including arthrocentesis). The objective will be to avoid development of synovitis and hemophilic arthropathy. For the management of chronic synovitis, we advise radiosynovectomy as the first-line procedure. If, after three procedures with 6-month interval, radiosynovectomy fails, an arthroscopic synovectomy must be indicated. Many hemophilic patients develop hemophilic arthropathy in the second to fourth decades. Then possible treatments include arthroscopic joint debridement and total joint arthroplasty [29].

\section{References}

1. Lafeber FP, Miossec P, Valentino LA (2008) Valentino LA Physiopathology of haemophilic arthropathy. Haemophilia14: 3-9.

2. Manco-Johnson MJ, Abshire TC, Shapiro AD, Riske B, Hacker MR, et al. (2007) Prophylaxis versus episodic treatment to prevent Joint Disease in Boys with Severe Hemophilia. N Engl J Med 357:535-544.

3. Nilsson IM, Berntorp E, Lofqvist T, Pettersson H (1992) Twenty-five years experience of prophylactic treatment in severe haemophilia A and B. J Int Med 232: 25-32.

4. Hilgartner MW (2002) Current treatment of hemophilic arthropathy. Curr Opin Pediatr 14: 46-49.

5. Jansen NW, Roosendaal G, Bijlsma JW, Degroot J, Lafeber FP (2007) Exposure of human cartilage tissue to low concentrations of blood for a short period of time leads to prolonged cartilage damage: an in vitro study. Arthritis Rheum 56: 199-207.

6. Valentino LA, Hakobyan N (2006) Histological changes in murine haemophilic synovits: a quantitative grading system to assess blood-induced synovitis. Haemophilia 12: 654-662.

7. Valentino LA, Hakobyan N, Rodriguez N, Hoots WK (2007) Pathogenesis of haemophilic synovitis: experimental studies on blood-induced joint damage. Haemophilia 13:10-13.

8. Hooiveld MJ, Roosendaal G, Jacobs KM, Vianen ME, van den Berg HM, et al. (2004) Initiation of degenerative joint damage by experimental bleeding combined with loading of the joint. Arthritis \& Rheumatism 50: 2024-2031.

9. Hakobyan N, Kazarian T, Jabbar AA, Jabbar KJ, Valentino LA (2004) Pathobiology of hemophilic synovitis I: overexpression of $\mathrm{mdm} 2$ oncogene. Blood 104: 2060-2064.

10. Valentino LA, Hakobyan N, Kazarian T, Jabbar KJ, Jabbar AA (2004) Experimental haemophilic synovitis: rationale and development of a murine model of human factor VIII deficiency. Haemophilia 10: 280-287.

11. Acharya SS, Schloss R, Dyke JP, Myntz DN, Christos P, et al. (2008) Powe Doppler sonography in the diagnosis of hemophilic synovitis - a promising tool. J Thromb Haemost 6: 2055-2061.

12. Merchan ECR, De Orbe A, Gago J (1992) Ultrasound in the diagnosis of early stages of hemophilic arthropathy of the knee. Acta Orthop Belg 58: 122-125.

13. Wallny T, Brackmann HH, Semper H, Schumper G, Effenberger W, et al. (2000) 
Intra-articular hyaluronic acid in the treatment of haemophilic arthropathy of the knee. Clinical, radiological and sonographical assessment. Haemophilia 6: 566570 .

14. Klukowska A, Zcirny Z, Laguna P, Brzewski M, Serafin-Krol MA, et al. (2001) Correlation between clinical, radiological and ultrasonographical image of knee joints in children with haemophilia. Haemophilia 7: 286-292

15. Querol F, Rodriguez-Merchan EC (2011) The role of ultrasonography in the diagnosis of the musculo-skeletal problems of haemophilia. Haemophilia [Epub ahead of print]

16. Rattray B, Nugent DJ, Young G (2006) Celecoxib in the treatment of haemophilic synovitis, target joints, and pain in adults and children with haemophilia. Haemophilia 12: 514-517.

17. De la Corte-Rodriguez H, Rodriguez-Merchan EC, Jimenez-Yuste V (2011) Radiosynovectomy in patients with chronic haemophilic synovitis: when is more than one injection necessary? Europ J Haematol 86: 430-435

18. De la Corte-Rodriguez H, Rodriguez-Merchan EC, Jimenez-Yuste V (2011) Radiosynovectomy in Haemophilia: Quantification of its Effectiveness through the Assessment of 10 Articular Parameters. J Thromb Haemost 9: 928-935.

19. De la Corte-Rodriguez H, Rodriguez-Merchan EC, Jimenez-Yuste V (2011) What patient, joint and isotope characteristics influence the response to radiosynovectomy in patients with haemophilia? Haemophilia 17: e990-e998.

20. Rodriguez-Merchan EC, Wiedel JD (2001) General principles and indications of synoviorthesis (medical synovectomy) in haemophilia. Haemophilia 7: 6-10.

21. Rodriguez-Merchan EC, Luck JV Jr, Silva M (2003) Synoviorthesis in haemophilia. In: The Haemophilic Joints-New Perspectives. Blackwell, Oxford.

22. Roberts HR, Monroe DM, White GC (2004) The use of recombinant factor VIla in the treatment of bleeding disorders. Blood 104: 3858-64.

23. Mortazavi SMJ, Asadollahi S, Farzan M, Shahriaran S, Aghili M, et al. (2007) ${ }^{32} \mathrm{P}$ colloid radiosynovectomy in treatment of chronic haemophilic synovitis: Iran experience. Haemophilia 13: 182-188.

24. Kavakli K, Aydogdu S, Taner M, Duman Y, Balkan C,et al. (2008) Radioisotope synovectomy with rhenium 186 in hemophilic synovitis for elbows, ankles and shoulders. Haemophilia 14: 518-523.

25. Rodriguez-Merchan EC, Quintana M, De la Corte-Rodriguez H, Coya J (2007) Radioactive synoviorthesis for the treatment of haemophilic synovitis. Haemophilia 13: 32-37.
26. Corrigan JJ, Damiano MI, Leissinger C, Wulff K (2002) Treatment of chronic haemophilic synovitis in humans with D-penicillamine. Haemophilia 9: 64-68.

27. Radossi P, Baggio R, Petris U, De Biasi F, Risato R, Davoli PG, Tagariello G (2003) Intra-articular ryfamicin in haemophilic arthropathy. Haemophilia 9: 60-63.

28. Rodriguez-Merchan EC, Wiedel JD, Wallny T, Hvid I, Berntorp E, et al. (2003) Elective orthopaedic surgery for inhibitor patients. Haemophilia 9: 625-631

29. Fernandez-Palazzi F, Viso R, Bernal R (2003) Oxytetracycline clorhydrate as a new material for chemical synoviorthesis in haemophilia. In: The Haemophilic Joints-New Perspectives. Blackwell, Oxford.

30. Rodriguez Merchan EC, Magallon M, Galindo E (1994) Joint debridement for haemophilic arthropathy of the knee. Int Orthop 18: 135-138.

31. Sheth DS, Oldfield D, Ambrose C, Clyburn T (2004) Total knee arthroplasty in hemophilic arthropathy. J Arthroplasty 19: 56-60.

32. Rodriguez-Merchan EC, Luck JV Jr, Silva M, Riera JA, Wiedel JD, et al. Tota knee replacement in the haemophilic patient. In: The Haemophilic Joints. New Perspectives. Blackwell Publishing, Oxford.

33. Rodriguez-Merchan EC (2002) Total knee arthroplasty in patients with haemophilia who are HIV-positive. J Bone Joint Surg Br 84: 170-172.

34. Norian JM, Ries MD, Karp S, Hambleton J (2002) Total knee arthroplasty in hemophilic arthropathy. J Bone Joint Surg Am 84:1138-1141.

35. Ragni MV, Crossett LS, Rendón JH (1995) Postoperative infection following orthopaedic surgery in human immunodeficiency hemophiliacs with CD4 counts $<$ or $=200 / \mathrm{mm}^{3}$. J Arthroplasty 10: 716-721.

36. Powell DL, Whitener CJ, Dye CE, Ballard JO, Shaffer ML, et al. (2005) Knee and hip arthroplasty infection rates in persons with hemophilia: a 27 year single center experience during the HIV epidemic. Haemophilia 11: 233-239.

37. McDonald AG, Yang K, Roberts HR, Monroe DM, Hoffman M (2008) Perivascular tissue factor is down-regulated following cutaneous wounding: implications fo bleeding in hemophilia. Blood. 111: 2046-2048

38. McDonald A, Hoffman M, Hedner U, Roberts HR, Monroe DM (2007) Restoring hemostatic thrombin generation at the time of cutaneous wounding does not normalize healing in hemophilia B. J Thromb Haemost 5:1577-5183.

39. Rodriguez-Merchan EC. Aspects of current management: orthopaedic surgery in haemophilia. Haemophilia [Epub ahead of print]. 